\title{
Diagnostic thresholds for free light chains in multiple myeloma depend on the assay used
}

\author{
Xavier Bossuyt $t^{1,2} \cdot$ Michel Delforge $^{3} \cdot$ Martin Reynders $^{1} \cdot$ Doreen Dillaerts $^{2} \cdot$ Ben Sprangers $^{4} \cdot$ Karel Fostier $^{5}$. \\ Koen Poesen ${ }^{1,6} \cdot$ Martine Vercammen 7,8
}

Received: 29 October 2017 / Accepted: 9 November 2017

(c) Macmillan Publishers Limited, part of Springer Nature 2018

Free light chains (FLCs) are helpful markers for the diagnosis, prognosis, and follow-up of patients with monoclonal gammopathy [1-5]. Freelite $\kappa$ and $\lambda$ reagents (The Binding Site) were the first commercially available reagents to measure FLC in serum [6]. These reagents are based on polyclonal antibodies raised in sheep [6]. The clinical relevance of Freelite has been extensively demonstrated [15]. Since 2011, Siemens offers the N Latex test, which is based on monoclonal antibodies, for FLC measurement [7]. Monoclonal reagents have the advantage of high lot-to-lot consistency and reproducibility. A possible disadvantage is that epitopes can be missed [8]. N Latex FLC results differ in absolute values from Freelite results [7].

Electronic supplementary material The online version of this article (https://doi.org/10.1038/s41375-018-0041-0) contains supplementary material, which is available to authorized users.

Xavier Bossuyt

Xavier.Bossuyt@uzleuven.be

1 Laboratory Medicine, Immunology, University Hospitals Leuven, Leuven, Belgium

2 Department of Microbiology and Immunology, Experimental Laboratory Immunology, Catholic University of Leuven, Leuven, Belgium

3 Department of Hematology, University Hospitals Leuven, Leuven, Belgium

4 Department of Nephrology, University Hospitals Leuven, Leuven, Belgium

5 Department of Hematology, University Hospital VUB, Brussels, Belgium

6 Department of Neurosciences, Laboratory for Molecular Neurobiomarker Research, KU Leuven, Leuven, Belgium

7 Laboratory of Hematology, Universitair Ziekenhuis Brussel VUB, Brussels, Belgium

8 Present address: Department of Laboratory Medicine, AZ Sint-Jan, Brugge, Belgium
Given these differences it is not clear whether diagnostic and prognostic thresholds proposed for Freelite also hold for N Latex. For example, in AL amyloidosis a baseline difference between involved and uninvolved light chain (dFLC) of $180 \mathrm{mg} / \mathrm{L}$ by Freelite corresponded to $165 \mathrm{mg} / \mathrm{L}$ by $\mathrm{N}$ Latex for survival and response to therapy was predicted by $>50 \%$ decrease in Freelite dFLC or $>33 \%$ decrease in N Latex dFLC [9].

Recently, the International Myeloma Working Group included FLC as a biomarker of malignancy and defined involved/uninvolved FLC ratio $>100$ (on the condition that involved FLC $>100 \mathrm{mg} / \mathrm{L}$ ) as a multiple myeloma (MM) event [10]. Such event is considered an indication for treatment. This criterium was based on results obtained with Freelite [11]. We addressed whether thresholds for MM defined with Freelite can be used for N Latex by directly comparing both FLC assays in patients with MM and monoclonal gammopathy of undetermined significance (MGUS).

Samples from $205 \mathrm{MM}$ patients (131 males, 74 females) (median age 66 years; age range: $34-88)$ (88 IgGк, 26 $\operatorname{IgG} \lambda$, $28 \operatorname{IgA\kappa }, 19 \operatorname{IgA} \lambda, 1 \operatorname{IgM\kappa }, 1 \operatorname{IgM} \lambda, 22 \kappa, 19 \lambda$, and 1 no clear identification) were included: 191 were from the University Hospitals Leuven and 14 from the University hospital Brussel. For 159 patients, the sample was obtained close to the diagnosis ( $<3$ months). In addition, 525 samples from 255 different MGUS patients were included (141 males, 114 females) (median age 67 years; age range 34-93) (103 $\operatorname{IgG\kappa }, 74 \operatorname{IgG} \lambda$, $16 \operatorname{Ig} A \kappa, 15 \operatorname{Ig} A \lambda, 1 \operatorname{Ig} A$ type?, $10 \operatorname{IgM\kappa }, 16 \operatorname{IgM} \lambda, 5 \lambda, 11$ a combination of monoclonal proteins, 4 no clear identification) were included: 240 were from University Hospitals Leuven and 15 from University hospital Brussel VUB. Several of these patients had follow-up determinations (after $~ 2,4$ and 6 years); 97, 78,48 , and 32 patients had, respectively, 1, 2, 3, and 4 determinations.

All samples were run on the Optilite system using polyclonal Freelite reagents (The Binding Site) and on a BN 
Fig. 1 Scatter plot of $\kappa / \lambda$ ratio by $\mathrm{N}$ Latex ( $x$-axis) and Freelite (yaxis) in patients with MGUS (blue open symbols) and in patients with MM (orange filled symbols). Dotted line represents line of equality. The spearman rank correlation coefficient was 0.917 (95\% CI: 0.904-28)

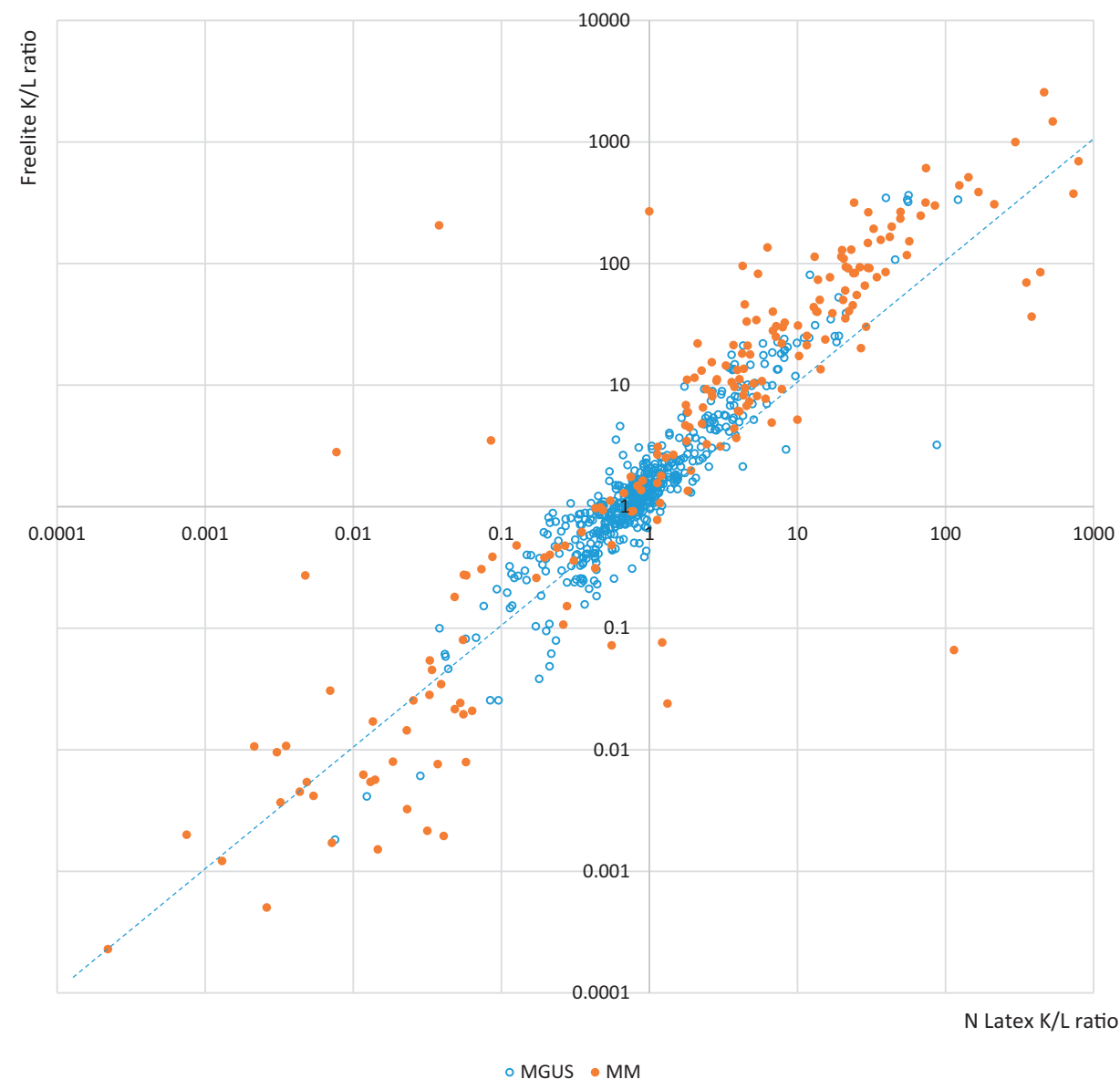

-MGUS - MM
Prospec instrument using monoclonal $\mathrm{N}$ Latex reagents (Siemens) by the manufacturer according to their instructions. The study was approved by the local ethics committee (Leuven: ML10187 / Brussels: B.U.N.143201421779).

Overall, $\kappa$ FLC were higher by Freelite than by N Latex (Suppl data Fig. 1). Values for $\lambda$ (Spearman correlation coefficient rs: 0.855$)$ were more scattered between the two methods than values for $\kappa$ (rs: 0.941). For low $\lambda$ FLC values ( $<7 \mathrm{mg} / \mathrm{L}$ by Freelite), results obtained by Freelite were lower than results obtained by $\mathrm{N}$ latex. The distribution of $\lambda$ FLC values showed a discontinuous distribution for Freelite at a level of $7 \mathrm{mg} / \mathrm{L}$, which is related to a switch in serum dilution (1:2 versus 1:8)] (Suppl data Fig. 1).

The $\kappa / \lambda$ ratio is shown in Fig. 1 and shows a higher ratio for Freelite than for $N$ Latex for values $>1$ (by Freelite). This was not the case for values $<1$.

All samples with an involved/uninvolved FLC ratio $>$ 100 by Freelite had involved FLC $>100 \mathrm{mg} / \mathrm{L}$ by Freelite (data not shown). Next, we evaluated the concordance between Freelite $\kappa / \lambda$ ratio thresholds of $\geq 100$ (for $\kappa$ monoclonals) and $\leq 0.01$ (for $\lambda$ monoclonals) with several $N$ Latex $\kappa / \lambda$ ratio thresholds (between 20 and 100 and between 0.01 and 0.08 for $\kappa$ and $\lambda$ monoclonals, respectively). The Kappa statistics are summarized in Fig. 2 and show that the best
Kappa (interobserver) statistic was found with $\mathrm{N}$ Latex $\kappa / \lambda$ ratio thresholds $\geq 30$ and $\leq 0.03 / \leq 0.02$.

For example, of the 39 patients having a $\kappa / \lambda$ FLC ratio $>$ 100 with Freelite, only 11 had such high ratio with N Latex. On the other hand, four patients had a $\kappa / \lambda$ FLC ratio $>100$ with $N$ Latex but not with Freelite. Changing the $\kappa / \lambda$ ratio threshold from 100 to 30 for N Latex resulted in 29 patients with abnormal FLC ratio with both assays, 10 with abnormal ratio with Freelite and 8 with abnormal ratio with $\mathrm{N}$ Latex. Twenty three patients had a $\kappa / \lambda$ FLC ratio $<0.01$ with Freelite. Of these, only 11 also had such low ratio with $\mathrm{N}$ Latex. On the other hand, five patients had a $\kappa / \lambda$ FLC ratio $<0.01$ with $N$ Latex but not with Freelite. Changing the $\kappa / \lambda$ ratio threshold from 0.01 to 0.03 for $\mathrm{N}$ Latex resulted in 19 patients with abnormal FLC ratio with both assays, 4 with abnormal ratio with Freelite and 8 with abnormal ratio with $\mathrm{N}$ Latex. All samples with an $\kappa / \lambda$ FLC ratio $>30$ or $<0.03$ by $\mathrm{N}$ Latex had involved FLC $>100 \mathrm{mg} / \mathrm{L}$ by N Latex (data not shown).

The majority of samples with an involved/uninvolved FLC ratio $>100$ in our study were from MM patients (with CRAB features, i.e., hypercalcemia, renal failure, anemia, bone lesions). The non-MM patients included one patient with a smoldering MM who developed MM 3 months later 

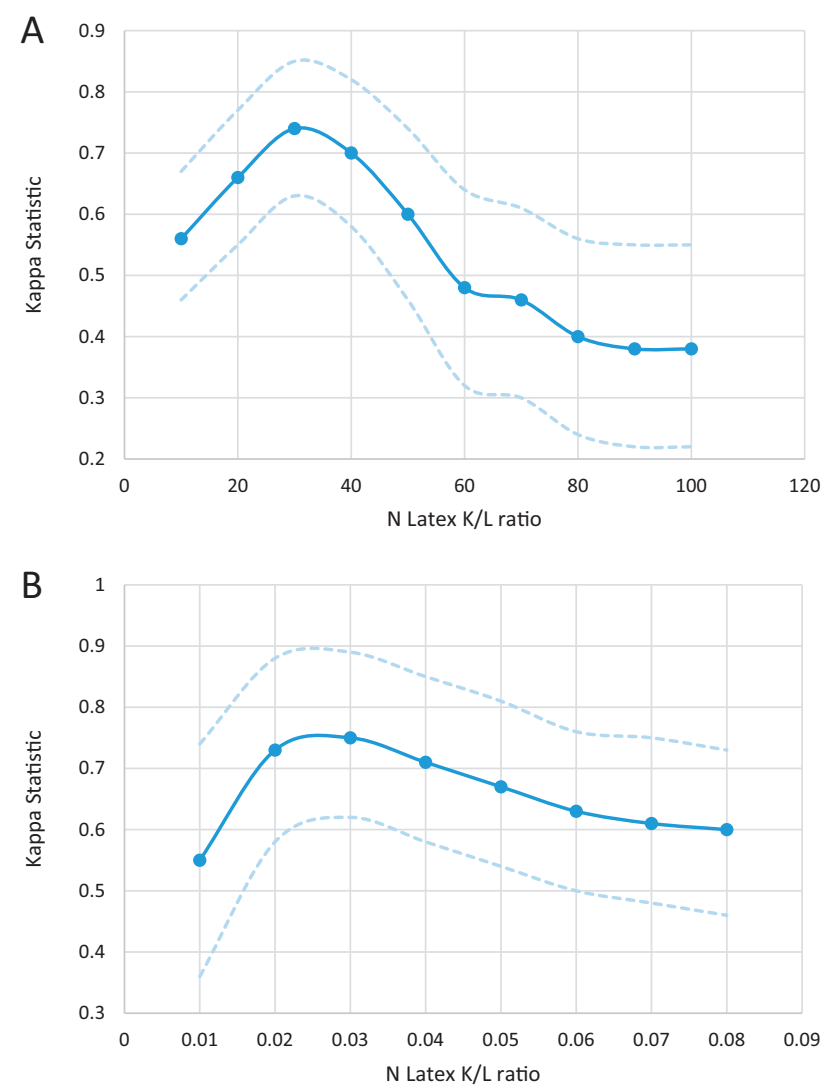

Fig. 2 Kappa statistic for interobserver agreement. $\kappa$ and $\lambda$ FLC was determined in patients with MM and in patients with MGUS with Freelite and with N Latex. a Kappa statistic for Freelite $\kappa / \lambda$ ratio $\geq 100$ and $N$ Latex $\kappa / \lambda$ ratio $\geq 20, \geq 30, \geq 40, \geq 50, \geq 60, \geq 70, \geq 80, \geq 90$, or $\geq 100$. b Kappa statistic for Freelite $\kappa / \lambda$ ratio $\leq 0.01$ and $N$ Latex $\kappa / \lambda$ ratio $\leq 0.01, \leq 0.02, \leq 0.03, \leq 0.04, \leq 0.05, \leq 0.06, \leq 0.07$, or $\leq 0.08$. Dotted lines represent $95 \%$ confidence interval

and three MGUS patients. The first MGUS patient had a $\kappa / \lambda$ FLC of 0.006 by Freelite and 0.028 by $\mathrm{N}$ Latex. He developed MM 6.5 years later. The second MGUS patient had several FLC determinations between 2007 and 2013. The $\kappa / \lambda$ varied between 321 and 364 by Freelite and between 55 and 121 by N Latex. He remained MGUS until the last follow-up in 2015 (thus over a 7-year period). The third MGUS patient had a $\kappa / \lambda$ ratio of 108 by Freelite and 46 by N Latex (in 2014). Three years later (2017), he still was MGUS (no CRAB features) ( $\kappa / \lambda$ ratio Freelite: 95.6). Thus, in these three MGUS patients, an involved/uninvolved FLC ratio $>100$ by Freelite (and involved FLC > $100 \mathrm{mg} / \mathrm{L}$ ) did not predict progression to MM (with CRAB features) within the first 2 years.

In conclusion, an analytical comparison of $\kappa / \lambda$ ratios measured by Freelite and by N Latex in MGUS and MM patients revealed that the $\kappa / \lambda$ ratios differ between Freelite and N latex. Diagnostic FLC thresholds for MM proposed for Freelite do not apply to $\mathrm{N}$ Latex. Efforts should be undertaken to align/harmonize clinical interpretation between the two assays. Additional clinical studies are needed to establish the predictive value of proposed $\kappa / \lambda$ ratio thresholds in MGUS patients for progression to MM.

Acknowledgements We thank Siemens and The Binding site for providing the reagents and for performing the assays. We thank Oscar Berlanga and Stephen Harding (both from the Binding Site) and Carola Wagner (from Siemens) for helpful discussions.

Author contribution $\mathrm{XB}$ and $\mathrm{MV}$ designed the study and wrote the paper. XB, MV, MR, and DD analyzed the data. MD, KF, and BS take care of the patients with multiple myeloma and revised the manuscript. KP discussed the data and critically reviewed the study and the manuscript.

\section{Compliance with ethical standards}

Conflict of interest MV, KP, and $\mathrm{XB}$ received travel fees from The Binding Site. BS received research support from The Binding Site. The remaining authors declare no conflict of interest.

\section{References}

1. Kyle RA, Rajkumar SV. Criteria for diagnosis, staging, risk stratification, and response assessment of multiple myeloma. Leukemia. 2009;23:3-9.

2. Rajkumar SV, Kyle RA, Therneau TM, Melton LJ 3rd, Bradwell AR, Clark RJ, et al. Serum free light chain ratio is an independent risk factor for progression in monoclonal gammopathy of undetermined significance. Blood. 2005;106:812-7.

3. Vermeersch P, Vercammen M, Holvoet A, Vande Broek I, Delforge $\mathrm{M}$, Bossuyt X. Use of interval-specific likelihood ratios improves clinical interpretation of serum FLC results for the diagnosis of malignant plasma cell disorders. Clin Chim Acta. 2009;410:54-8.

4. Palladini G, Russo P, Bosoni T, Verga L, Sarais G, Lavatelli F, et al. Identification of amyloidogenic light chains requires the combination of serum-free light chains and with immunofixation of serum and urine. Clin Chem. 2009;55:499-504.

5. Palladini G, Dispenzieri A, Gertz MA, Kumar S, Wechalekar A, Hawkins PN, et al. New criteria for response to treatment in immunoglobulin light chain amyloidosis based on free light chain measurement and cardiac biomarkers: impact on survival outcomes. J Clin Oncol. 2012;30:4541-9.

6. Bradwell AR, Carr-Smith HD, Mead GP, Tang LX, Showell PJ, Drayson MT, et al. Highly sensitive, automated immunoassay for immunoglobulin free light chains in serum and urine. Clin Chem. 2001;47:673-80.

7. te Velthuis H, Knop I, Stam P, et al. N Latex FLC - new monoclonal high-performance assays for the determination of free light chain kappa and lambda. Clin Chem Lab Med. 2011;49:1323-32.

8. Carr-Smith HD, Jenner EL, Evans JAR, Harding SJ. Analytical issues of serum free light chain assays and the relative performance of polyclonal and monoclonal based reagents. Clin Chem Lab Med. 2016;54:997-1003.

9. Palladini G, Jaccard A, Milani P, et al. Circulating free light chain measurement in the diagnosis, prognostic assessment and evaluation of response of AL amyloidosis: comparison of Freelite and N latex FLC assays. Clin Chem Lab Med. 2017;55:1734-43.

10. Rajkumar SV, Dimopoulos MA, Palumbo A, Blade J, Merlini G, Mateos MV, et al. International Myeloma Working Group updated criteria for the diagnosis of multiple myeloma. Lancet. 2014;15:e538-48.

11. Larsen JT, Kumar SK, Dispenzieri A, Kyle RA, Katzmann JA, Rajkumar SV Serum free light chain ratio as a biomarker for highrisk smoldering multiple myeloma. Leukemia. 2013;27:941-6 